\title{
Effects of Filler Wire Intervention on Gas Tungsten Arc: Part I - Mechanism
}

\author{
A novel sensing method of detecting voltage signals between a filler wire \\ and tungsten electrode/workpiece was proposed
}

BY S. ZOU, Z. WANG, S. HU, G. ZHAO, W. WANG, AND Y. CHEN

\begin{abstract}
For gas tungsten arc welding (GTAW), the effects of filler wire on the GTA are worth being clarified, which will help deepen the understanding of arc characteristics and inspire new ideas for the real-time monitoring of weld quality. To this end, this work proposed a novel sensing method of detecting probe voltages (i.e., the voltage signals between a filler wire and tungsten electrode/workpiece). Based on this method, in this first part of the work, a tungsten probe was used to replace the filler wire and to interact with the arc in the specific experiments to elucidate the static and dynamic interaction mechanisms between the GTA and filler wire. The results showed that the filler wire intervention deflects the arc to various degrees and will change the voltage signals. As a metal conductor, the filler wire will increase the arc voltage by increasing the average electric field strength. However, its effects on the different areas of the arc are not always consistent, which makes the change trend of the probe voltages not always the same. Moreover, due to thermal inertia, the probe voltage does not strictly change synchronously with the arc voltage under the dynamic disturbance. This work lays a theoretical foundation for monitoring the stability of the GTAW process.
\end{abstract}

\section{KEYWORDS}

- Arc Characteristics • Arc Voltage • Arc Sensing

- Gas Tungsten Arc (GTA) • Filler Wire • Gas Tungsten Arc Welding (GTAW)

\section{Introduction}

Gas tungsten arc welding (GTAW) is one of the precision welding techniques for obtaining high-quality weldments. It can weld a variety of industrial structural metals and is widely used in aerospace, atomic energy, pressure vessels, and other industrial fields. Due to its wide application, the quality assurance of GTAW is particularly important. However, even with optimized welding parameters, it is still difficult to guarantee weld quality with automated GTAW in complex environments because of its uncertainty and time variation. This situation requires the development of on-line monitoring and control technologies for weld quality.

The geometry of the weld pool is closely related to the realtime welding state and largely determines the formation of welds after solidification. Therefore, the accurate extraction of weld pool geometry will help to understand and then monitor the dynamic welding process, and it will also contribute to providing useful feedback for the real-time control of weld quality. The extraction of weld pool geometry is often based on visual sensing techniques. Chen et al. used passive visual sensing to extract the distance between the tungsten electrode tip and its reversed reflection in the weld pool under suitable exposure conditions, to characterize the height of the weld pool surface (its concavity/convexity) (Ref. 1). Fan et al. developed a threeway passive visual sensing system to simultaneously detect the weld pool geometry from both sides of the workpiece (Ref. 2). Taking laser as an auxiliary light source, Zhang and Song et al. reconstructed the weld pool by virtue of the designed 3D visual sensing system (Refs. 3-5), and based on this system, Liu and Dong et al. have extracted the geometric parameters of the 3D weld pool for modeling of weld-quality control (Refs. 6, 7).

In addition to the weld pool geometry, the dynamic behaviors of the weld pool have also received much attention. During autogenous GTAW, the weld pool is excited to oscillate by pulse current. The frequency and amplitude of weld pool oscillation can be used to describe the weld penetration status because both of them will change as the volume of the weld pool changes. When the solid restraint at the bottom of the weld pool disappears, the weld pool will have a sudden subsidence, and its oscillation frequency and amplitude will be abruptly changed accordingly. This abrupt change can be used to characterize the critical complete joint penetration status. These dynamic behaviors of the weld pool can be monitored by the arc voltage (Refs. 8-11) or the evolution of the laser pattern reflected from the weld pool surface in the structured-laser visual sensing system (Refs. 12-14).

Arc sound is also a noteworthy information source that can reflect the weld pool status. It can be roughly considered that the arc sound signal in pulsed GTAW is determined by the arc sound source and the modulating arc sound channel. The sound source is generated from the arc energy change, 


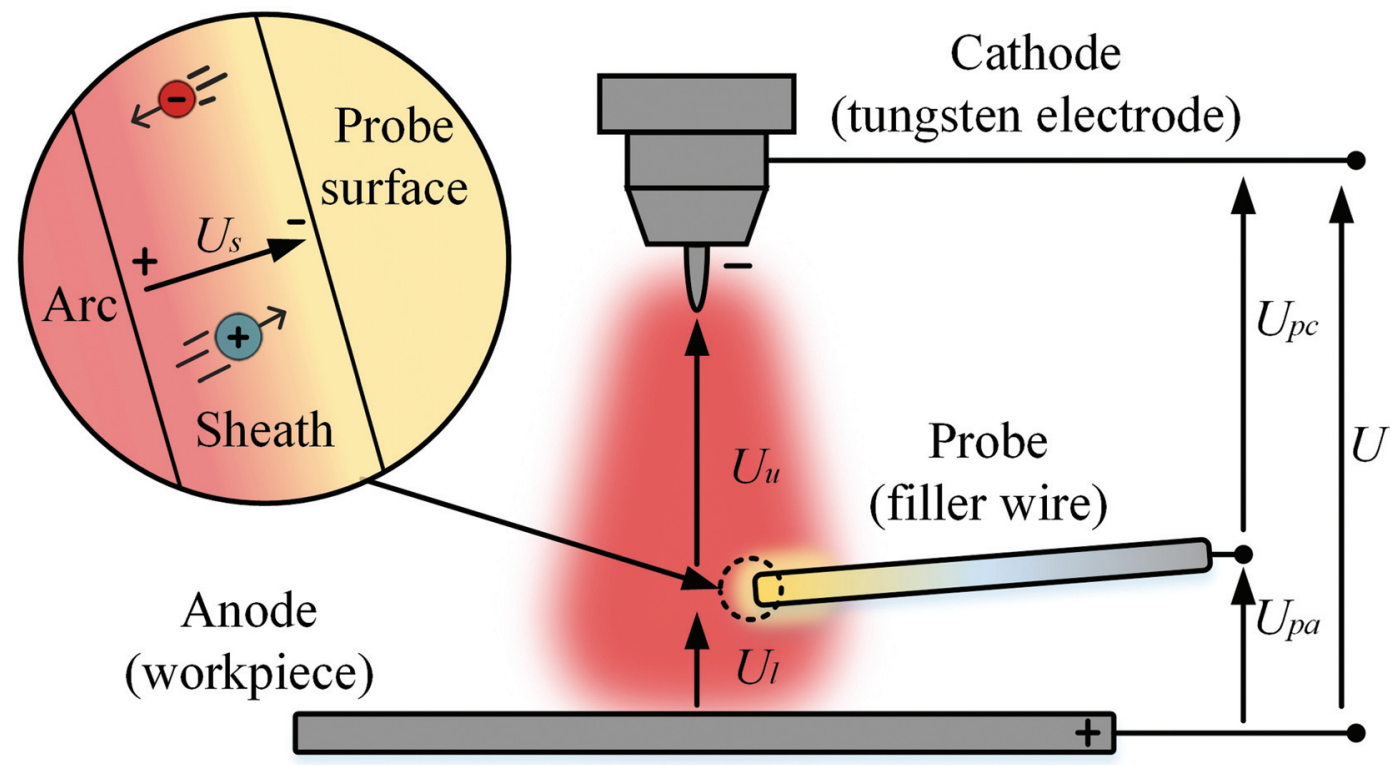

Fig. 1-Schematic diagram of the sensing principle.

and the sound channel can be considered as a cavity covered by the shielding gas atmosphere (Refs. 15-17). According to Lv et al. (Refs. 16,17), statistical characteristics of the arc sound in time domain can be used to distinguish different penetration statuses due to the difference in the arc energy, and the different penetration statuses will also result in different formant distribution of the sound channel because of the change in the sound channel state (e.g., an excessively penetrated weld pool will destroy the sound channel). Zhang et al. used the Fisher distance and principal component analysis to select the frequency components reflecting the weld penetration status in pulsed GTAW, then established a classification model and successfully distinguished the different weld penetration statuses (Ref. 18).

As mentioned above, so much valuable and potential information has been continuously mined, the relevant mechanisms have been deeply explained, and various sensing methods have been proposed to serve on-line monitoring and control of GTAW quality. However, the reported studies in the academic field have focused more on autogenous GTAW than GTAW with filler wire. Furthermore, researchers have devoted more attention to studies on weld pool status, often ignoring the effect of the liquid metal generated from the filler wire (if it is employed) on the process. Although the employment of the filler wire will expand the application of GTAW, it will also interfere with the GTA and impact the extraction of characteristic information about the weld pool (e.g., disturbing its regular oscillation frequency under pulse current), which is not conducive to the welding process stability and monitoring of the weld pool. Yudodibroto et al. studied the effects of filler wire on weld pool oscillation in GTAW and thought the oscillation frequency could still be measured to reflect the weld penetration status under the mode of uninterrupted metal transfer (Ref. 19), but the further study has not been reported yet; for instance, the interaction mechanisms between the filler wire and GTA are still unclear.
The purpose of this work is to clarify the effects of the filler wire intervention on the GTA. For this purpose, a novel sensing method of detecting the probe voltages (i.e., the voltage signals between the filler wire and tungsten electrode/workpiece) was proposed. Moreover, this work was split into two parts for elaboration. This first part aims at expounding the principal interaction mechanisms between the GTA and filler wire, and the second part will discuss the behaviors of liquid droplets. In the first part, to simplify the study, a tungsten probe was used to replace the filler wire and to interact with the arc through specific experiments. This work helped to enhance the understanding of electrical arc characteristics and laid the foundation for on-line monitoring and control of the GTAW quality when the filler wire was employed.

\section{Sensing Methodology}

In the autogenous GTAW process, electrical arc is established between the nonconsumable tungsten electrode tip and liquid weld pool surface. Weld pool behaviors will change arc length and thus change arc voltage ( $U$, i.e., the voltage between the tungsten electrode and the workpiece). When the filler wire is introduced into the GTA, the feeding motion of the filler wire, the resulting metal transfer, and the pendant liquid droplets that oscillate at the end of the filler wire will affect the arc, and the filler wire itself as a suspended electrical conductor will also become an interference. The resultant interference signals will be coupled into the arc voltage, thereby obscuring the characteristic signals contained in the arc voltage that reflect the weld pool behaviors. It is difficult to distinguish their respective signal components and to clarify the interaction mechanisms between the filler wire and arc plasma with the arc voltage alone. Therefore, it may be possible to consider introducing additional sensing signals to help explain the relevant con- 


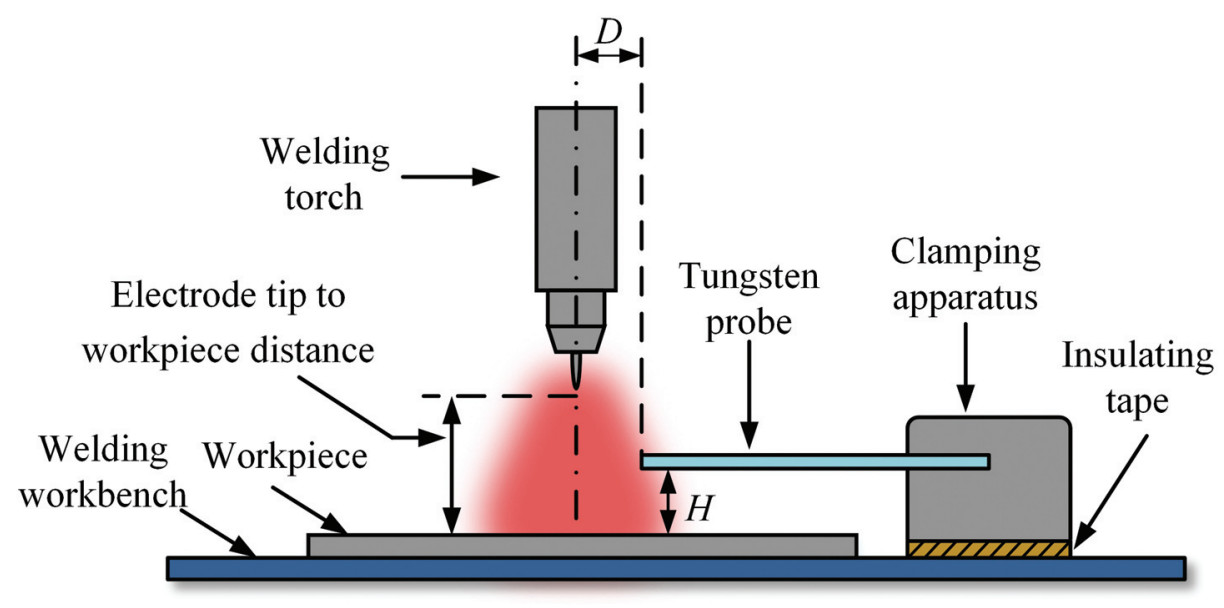

Fig. 2 - Experimental apparatus.

cerns.

Electrical arc is a self-sustaining discharge plasma whose electrical characteristics can be diagnosed by immersing metal probes (Ref. 20). Active (Ref. 21) or passive (Ref. 22) probe-sensing methods have also been used to monitor the keyhole process in plasma arc welding. Inspired by these, the filler wire can be regarded as a ready-made probe immersed in the arc atmosphere of GTAW, and probe voltages can be readily measured to provide more sensing information. Here, two probe voltages are detected, that is, the voltage between probe and tungsten electrode and the voltage between probe and workpiece, which are denoted as $U_{p c}$ and $U_{p a}$, respectively. It can be roughly thought that these two probe voltages divide the arc voltage into upper and lower parts. In addition to the voltage drop of the upper or lower arc column region (denoted as $U_{u}$ and $U_{l}$, respectively), these two probe voltages also contain the voltage drop of the cathode region $\left(U_{c}\right)$ and the voltage drop of the anode region $\left(U_{a}\right)$, respectively.

The number of positive and negative charges per unit volume at every moment is considered to be approximately equal in the GTA plasma, so the arc plasma is quasielectrically neutral. After the filler wire is introduced, electrons lighter than the positive ions will fly out of the arc plasma around the filler wire and flow to the surface of the filler wire at a faster rate, charging its surface to negative, relative to the arc plasma. As a result, a nonelectrically neutral transition layer, namely plasma sheath, will be formed between the arc plasma and the surface of the filler wire. Accordingly, the plasma sheath voltage is also implicit in the probe voltages. Although the sheath voltage $\left(U_{s}\right)$ may be very small, it is the plasma sheath that connects the filler wire to the arc plasma. A simplified schematic of the above description is demonstrated in Fig. 1, and the approximate relationship of these voltage signals can be expressed by Equation 1:

$$
\begin{aligned}
& U_{p a}=U_{a}+U_{l}+U_{s} \\
& U_{p c}=U_{c}+U_{u}-U_{s} \\
& U=U_{a}+U_{c}+U_{l}+U_{u}=U_{p a}+U_{p c}
\end{aligned}
$$

When $U$ is measured to reflect the overall electrical characteristics of GTA, $U_{p c}$ or $U_{p a}$ can be additionally used to reflect the local electrical characteristics of different regions of the GTA. Moreover, since the filler wire directly becomes a signal acquisition terminal, the probe voltages may be more suitable for characterizing the dynamic behaviors of the droplet at the end of the filler wire.

\section{Experimental System and Design}

\section{Experimental System}

The experimental system mainly consisted of the following three parts: welding system, high-speed camera system, and multichannel electrical signal acquisition system. The welding system was composed of a Fronius MagicWave 4000 GTAW power source (constant current mode), welding torch, and welding workbench. The Acuteye high-speed camera system equipped with optical dimmers and filters was used to provide auxiliary visual information about the arc shape. For the electrical signal acquisition system, electrical signals of interest were measured by Hall sensors and transmitted to a computer via a USB-4711A data acquisition card. The signal monitoring and recording was implemented by MATLAB ${ }^{\circledR}$ programming. The sampling frequency of electrical signals was $1024 \mathrm{~Hz}$. The original images were captured at a frame rate of 1024 frames per second and their resolution was $256 \times 256$ pixels.

\section{Experimental Design}

The experimental materials were Q235 mild steel plates with a dimension of $300 \times 60 \times 4 \mathrm{~mm}$ as the base metal and pure argon of $99.99 \%$ with a flow rate of $10 \mathrm{~L} / \mathrm{min}$ as the shielding gas. The diameter of the tungsten electrode was $2.4 \mathrm{~mm}$ and the length of the tungsten electrode protruding nozzle was $5 \mathrm{~mm}$. Bead-on-plate welding was employed in the flat position.

Based on the proposed sensing method, three groups of 


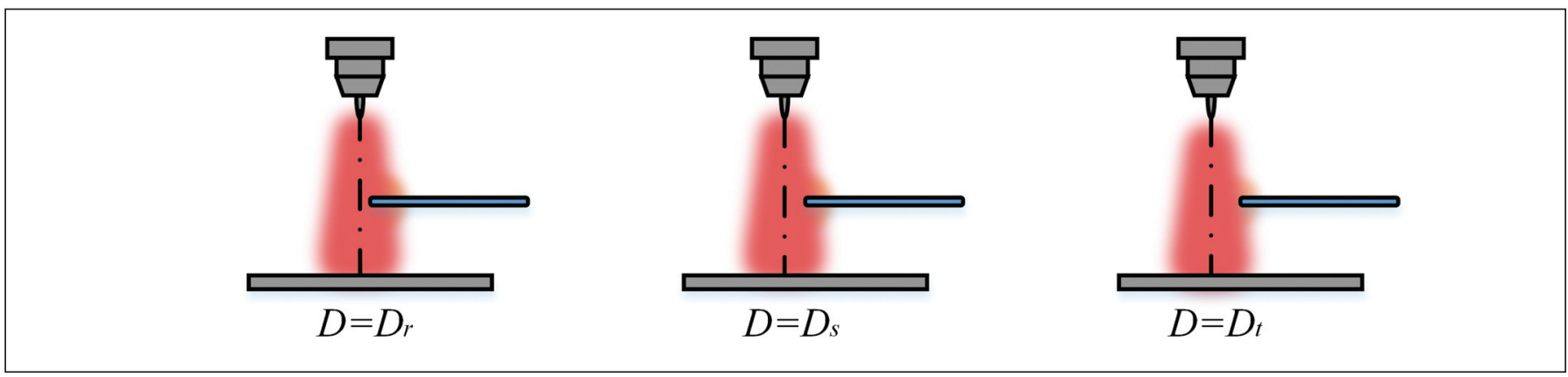

Fig. 3 - Diagram of relative position change $\left(0<D_{r}<D_{s}<D_{t}\right)$.

Table 1 - Experimental Parameters

\begin{tabular}{ccccccc} 
Experiment & $I(\mathrm{~A})$ & $v(\mathrm{~cm} / \mathrm{min})$ & ETWD* $(\mathrm{mm})$ & $H(\mathrm{~mm})$ & $D(\mathrm{~mm})$ & $\Delta T(\mathrm{~s})$ \\
\hline$\# 1$ & 65 & 7 & 7 & $3,2,1$ & - & - \\
$\# 2$ & 65 & 7 & 6.5 & 2 & $0,0.5$ & $7,6,5,4,3,2,1,0$ \\
$\# 3$ & 65 & 7 & 6 & 1.5 & - \\
\hline
\end{tabular}

*Note: ETWD means electrode tip to workpiece distance.

experiments (\#1, \#2, and \#3) have been designed and conducted. In all the experiments, a tungsten probe with a diameter of $2.4 \mathrm{~mm}$ was immersed into the arc atmosphere instead of the filler wire, and a relatively small welding current (I) was employed, which can ensure that the probe was not burnt as much as possible and the surface of the workpiece was slightly melted, thus avoiding the metal transfer and dynamic evolution behaviors of the weld pool. This way, the probe can simulate the filler wire to interfere with the arc plasma, thereby simplifying the objective of this study. Since the focus of this part of the work is only the inner interaction mechanisms between the GTA and filler wire, although this simulation is not equivalent to the actual filling process, it can also be considered that the effect of the filler wire as conductive metal on arc plasma follows similar mechanisms to that of the probe.

Figure 2 presents the schematic diagram of the experimental apparatus. As shown in Fig. 2, a tungsten probe was suspended parallel to the workpiece, and one end of this probe was fixed to the clamping apparatus. This clamping apparatus was placed on the welding workbench and isolated by nonconductive insulating tape. The axis of the welding torch was perpendicular to the workpiece surface. There are two positional parameters, $D$ and $H$, in Fig. 2, where $D$ is the horizontal distance between the probe tip and the symmetry axis of the electrode, and $H$ is the height difference between the probe and the workpiece surface. When the horizontal relative position of the symmetry axis of the electrode and the probe tip is as shown in Fig. 2, D is positive, and when the vertical relative position of the workpiece surface to the probe tip is as shown in Fig. 2, $H$ is positive.

The general experimental procedure was to keep the welding workbench stationary, suspend the probe at a height of $H$, and move the welding torch at a welding speed (v) in the horizontal direction to change $D$ ( $v$ is too small to affect the arc shape). When the absolute value of $D$ decreased, the direction of $v$ was considered positive, indicating that the welding torch and the probe were approaching.
The schematic relative position at different $D s$ can be seen in Fig. 3. During the experiments, arc voltage and probe voltage were collected, and the welding current was measured to ensure the voltage fluctuations were not caused by the current fluctuations. Experimental data was filtered by a simple moving average. Each average was calculated over a sliding window of 151 sample points, which was centered about the element in the current position.

The specific experimental designs and parameters are respectively shown in Fig. 4 and Table 1 . Experiment \#1 is mainly used to investigate the static effects of a probe on the GTA. As demonstrated in Fig. 4A, during Experiment $\# 1$, the welding torch moved horizontally several times to gradually go away from the probe tip until no probe voltage was detected. It moved $1 \mathrm{~mm}$ each time (e.g., $D_{a 2}-D_{a 1}=D_{a 1}$ $-D_{a 0}=1 \mathrm{~mm}$ ), and remained stationary for a while (10 s) after each movement. Experiment \#1 had been conducted at different $H s$, and in this way, the electrical signals can be obtained when the probe is in different spatial positions of the arc column.

Experiment \#2 aimed to study the dynamic effects of an intermittent/continuous reciprocating probe on the GTA. The design of Experiment \#2 is presented in Fig. 4B. The welding torch reciprocated equidistantly between position $D=0 \mathrm{~mm}$ and position $D=D_{b 0}=0.5 \mathrm{~mm}$. Whenever the welding torch was horizontally displaced from its original position to the new position in the same reciprocating motion mode, it stayed at the new position for a period of time $(\Delta T)$ and then proceeded to the next step. Different $\Delta T \mathrm{~s}$ (e.g., $\Delta T_{a}>\Delta T_{b}$ ) have been set during Experiment \#2. When $\Delta T$ was $0 \mathrm{~s}$, the probe generated a continuous interference, and when $\Delta T$ was a non-zero value, the probe caused an intermittent interference.

Experiment \#3 was about the dynamic effects of a continuously moving probe on the GTA at different positions. As shown in Fig. 4C, the welding torch reciprocated equidistantly several times between different horizontal positions to continuously generate dynamic interferences. The distance of 

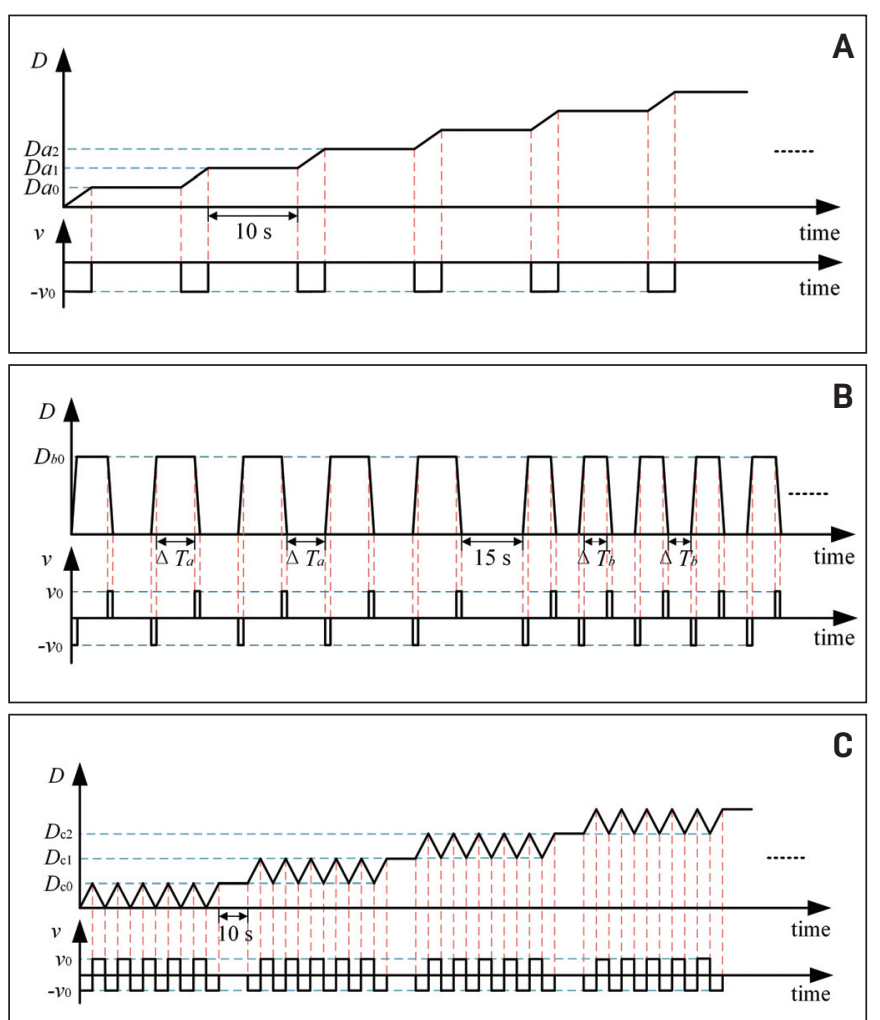

Fig. 4 - Experimental designs $\left(\mathrm{v}_{0}=7 \mathrm{~mm} / \mathrm{s}\right)$. $A$ - Experiment \#l; $B$ - Experiment \#2; C - Experiment \#3.

each movement was $1 \mathrm{~mm}$, and at intervals, the welding torch moved to a farther distance from the probe for a new reciprocating motion (e.g., $D_{c 2}-D_{c 1}=D_{c 1}-D_{c 0}=1 \mathrm{~mm}$ ).

\section{Results and Discussion}

\section{Static Effects}

Figure 5 displays the electrical signals measured in Experiment \#1, where $U_{p c}$ and $U_{p a}$ were measured separately. In Fig. 5, $D$ increases stepwise as time goes on. For ease of description, the process was roughly divided into phases $A, B$, and $C$, which respectively corresponded to the situation where the end of the probe was located in the original core area of the arc (the brightest area), the original noncore area of the arc, and the area outside the arc (no strict demarcation between the areas). The typical shapes of the core area of the arc can be seen in Fig. 6. Both the voltage signals and the arc shape were affected by the metal probe.

When the probe occupies the space area that originally belonged to the arc plasma, a plasma sheath will form near the probe surface and it will become a new partial edge area of the arc plasma. Accordingly, the overall arc shape will be changed. From phase A to B, $U$ drops stepwise and each drop resulted from an increase in $D$, while the growth in $D$ did not cause such a change in $U$ during phase $C$. Therefore, the metal conductor caused an increase in the arc voltage after it had immersed into an area of the GTA.

This can be explained from two aspects. On one hand, after the probe is directly inserted into the arc column, the conductive cross section in the vicinity of the probe is forcibly reduced, which destroys the conductive path with the minimum energy consumption under the original conditions, thus tending to change the direction of electron flow. Although the arc column will resist this change to keep the original conductive path as much as possible, the conductive path following the minimal energy principle will still tend to have different degrees of deflection (tend to become longer) under new conditions. On the other hand, the probe intervention will alter the thermal field of the arc column. Since the probe that is cold relative to the arc plasma increases the arc heat loss, the arc column will automatically shrink to reduce the contact area with the external medium, thereby minimizing the heat loss. It will also contribute to the reduction in the conductive cross section of the arc plasma near the probe, which will increase the average current density and thus increase the average electric field strength. In this way, the intervention of the metal probe causes the arc voltage to rise.

The relationship between the static probe at different positions and the stable arc voltage can be obtained by calculating the mean value of $U$ in the rectangular frames of Fig. $5 \mathrm{~A}, \mathrm{C}$, and $\mathrm{E}$, and the results are as shown in Fig. 7 . With the increase in $D, U$ declines more and more gently, and the increase in $H$ contributes to the rise of $U$. This is because the ionization in the core area of the arc is more sufficient, so the interference of the metal probe will gradually weaken as it moves away from the core region of the arc. In addition, the cathode is an electron emission source. The closer the probe is to the cathode, the greater its influence on the GTA will be. Therefore, the arc voltage will increase as $H$ rises.

It can be further found from Fig. 5 that whenever $D$ is changed once, $U$ can quickly level off after a small variation, while the changes in $U, U_{p c}$, and $U_{p a}$ are not completely consistent with one another. During phase A, when $U$ reaches a new stable level, $U_{p c}$ and $U_{p a}$ are still changing in opposite directions, and any of them has a greater variation than $U$. However, during phase $\mathrm{B}$, the variation in $U_{p c}$ and $U_{p a}$ caused by the change in $D$ becomes modest, and the trend of $U_{p a}$ in phase B is opposite to that in phase A. Also, the transition period of $U_{p c}$ and $U_{p a}$ becomes very short or even inconspicuous. In the final phase $C$, although $U$ is almost unaffected by the change in $D$, the change in $D$ will once again cause $U_{p c}$ and $U_{p a}$ to change significantly.

As mentioned earlier, the arc voltage signal can reflect the overall electrical characteristics of the arc, and the probe voltage signal can reflect the electrical characteristics of the local area of the arc. Such diverse variations in $U_{p c}$ and $U_{p a}$ indicate that the influence of the metal probe on the electrical characteristics of different areas of the arc at different phases is not always consistent. During phase A, although the arc above the probe maintained its original shape as much as possible, the lower part of the arc severely deflected. The larger the $H$ is, the more severe the deflection will be. This is because the electron flow needs to reform the shortest conductive path between the anode and cathode due to the physical barrier of the probe. As $H$ rises, the electron flow from the cathode will encounter this barrier earlier and thus be deflected more. In addition, the decrease in temperature around the probe tends to suppress the thermal ionization around the probe. Nevertheless, the neutral particles above the probe can still be directly bombard- 

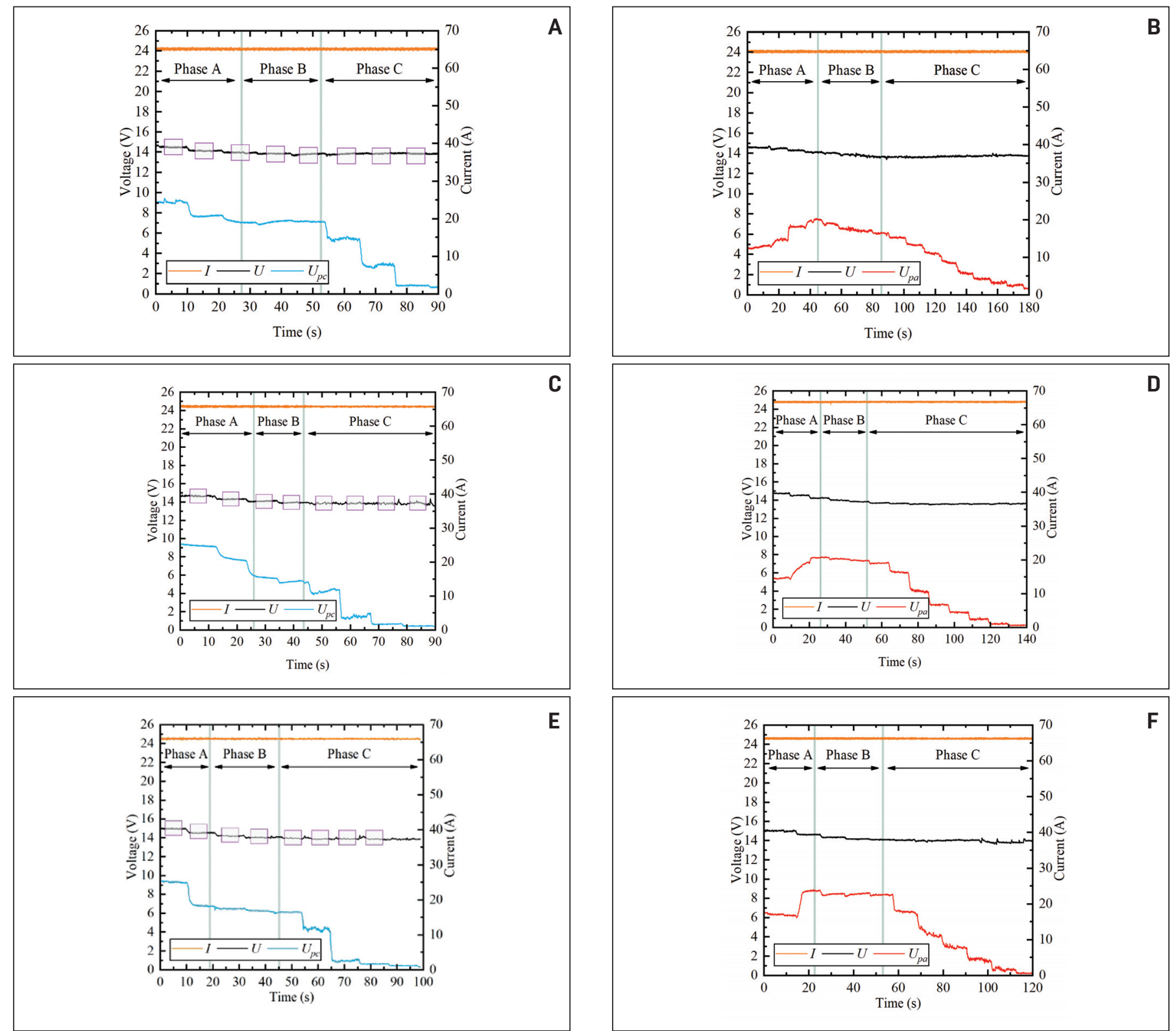

Fig. 5 - Electrical signals in Experiment \#l. $A-\mathrm{U}_{\mathrm{pc}}$ and $\mathrm{U}$ at $\mathrm{H}=1 \mathrm{~mm} ; \mathrm{B}-\mathrm{U}_{\mathrm{pa}}$ and $\mathrm{U}$ at $\mathrm{H}=1 \mathrm{~mm} ; \mathrm{C}-\mathrm{U}_{\mathrm{pc}}$ and $\mathrm{U}$ at $\mathrm{H}=2 \mathrm{~mm} ; \mathrm{D}-$ $\mathrm{U}_{\mathrm{pa}}$ and $\mathrm{U}$ at $\mathrm{H}=2 \mathrm{~mm} ; \mathrm{E}-\mathrm{U}_{\mathrm{pc}}$ and $\mathrm{U}$ at $\mathrm{H}=3 \mathrm{~mm} ; \mathrm{F}-\mathrm{U}_{\mathrm{pa}}$ and $\mathrm{U}$ at $\mathrm{H}=3 \mathrm{~mm}$.

ed by electrons from the cathode direction and thus be ionized. However, in the area below the probe, because of the physical block by the probe, it will be difficult for the neutral particles to be ionized by direct collision of electrons above the probe, and the originally charged ions will even be recombined due to insufficient energy. Therefore, during phase $\mathrm{A}$, the decrease in $D$ weakened the ionization of the lower part of the arc and then reduced the electric field, thus bringing $U_{p a}$ down, which corresponded to a weaker heat production capacity, although the arc length below the probe seemed to be longer. Meanwhile, the heat production capacity of the upper part of the arc was enhanced to meet the increased overall heat production capacity of the arc column, so the ionization degree and electric field intensity of the upper part of the arc increased, and $U_{p c}$ grew accordingly. In such a way, when the end of the probe is in the original core area of the arc, as $D$ increas- es, the main conductive path of the arc is gradually restored, the arc deflection is reduced, and the gap in ionization between the upper and lower parts of the arc is narrowed, so $U_{p a}$ rises and $U_{p c}$ falls. In phase $\mathrm{B}$, the end of the probe retreated to the original noncore area of the arc, and the main conductive path of the original core area was almost restored. The effects of the probe to the arc were weakened, and the probe no longer caused apparent arc deflection. The upper and lower parts of the arc presented as a whole, and the trend of $U_{p a}$ and $U_{p c}$ unified with that of $U$. During phase $C$, although the interference of the probe was not enough to change the arc voltage, the edge area of the arc still had a gradient distribution of unstable charges, so apparent changes in the probe voltages could still be detected.

Even if the physical medium forcibly intervenes in the core area of the arc, the arc can still self regulate in a short 


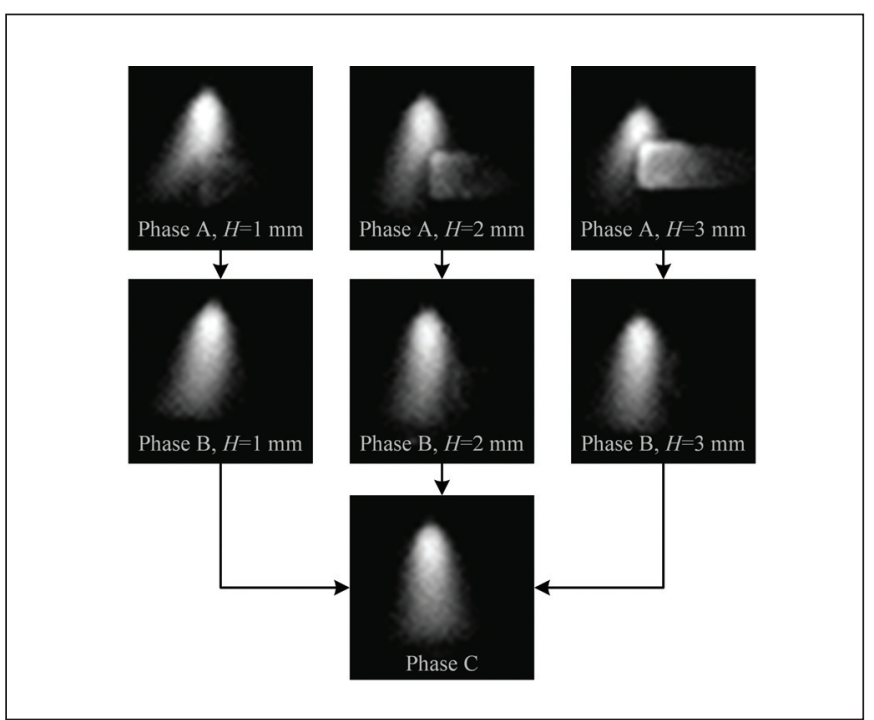

Fig. 6- Typical shapes of the core area of the arc from phase $A$ to $C$.

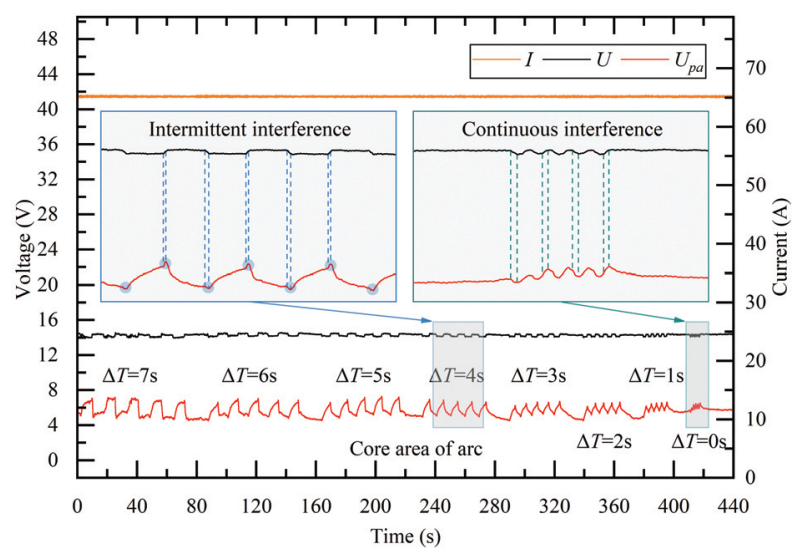

Fig. 8 - Electrical signals in Experiment \#2.

time to present a new state of overall stable heat production with the lowest energy consumption. However, in the area near the anode, as the probe interference deepens, the arc stiffness is not as good as before, and it tends to be unstable. It is conceivable that as the interference increases, the unstable area starting from the anode side will gradually expand toward the cathode side. Eventually, the arc will extinguish because it will fail to maintain a stable self-sustained discharge process.

In the actual welding process, the interaction between the filler wire and arc should conform to the above mechanisms, which will also cause the similar interference to the arc (except for metal transfer). To minimize the interference of the filler wire, it may be recommended to keep the end of the filler wire melted in phase B. This is because, on the one hand, its interference on the core area of the arc is small; on the other hand, if the end of the filler wire is melted in phase $C$, although the arc shape is stable, the filler wire may be melted intermittently due to the lack of heat. In addition, according to Equation 1, only one of the probe voltages,

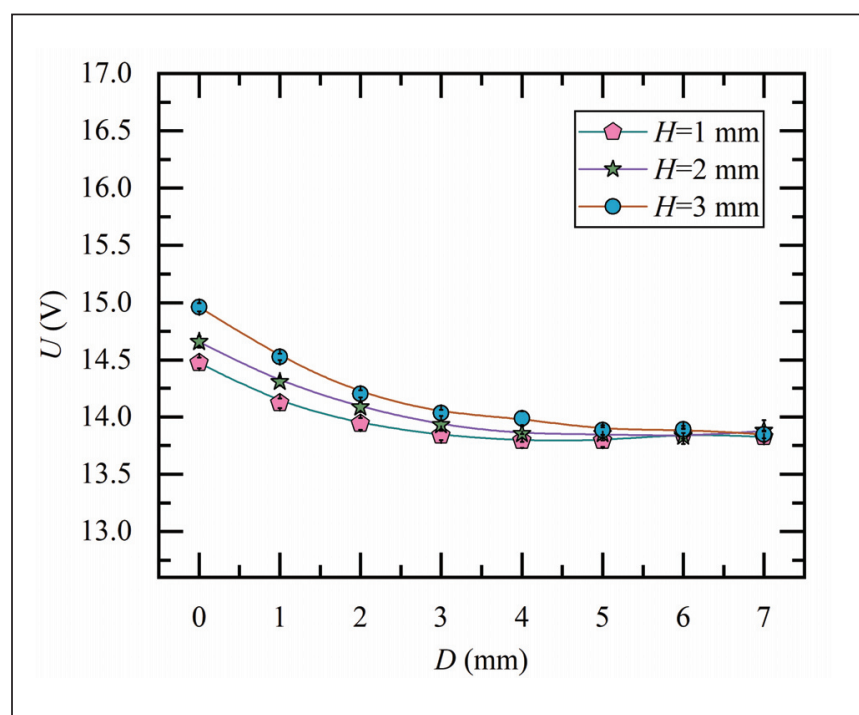

Fig. 7-Arc voltage with the static probe immersed in different positions of the arc column.

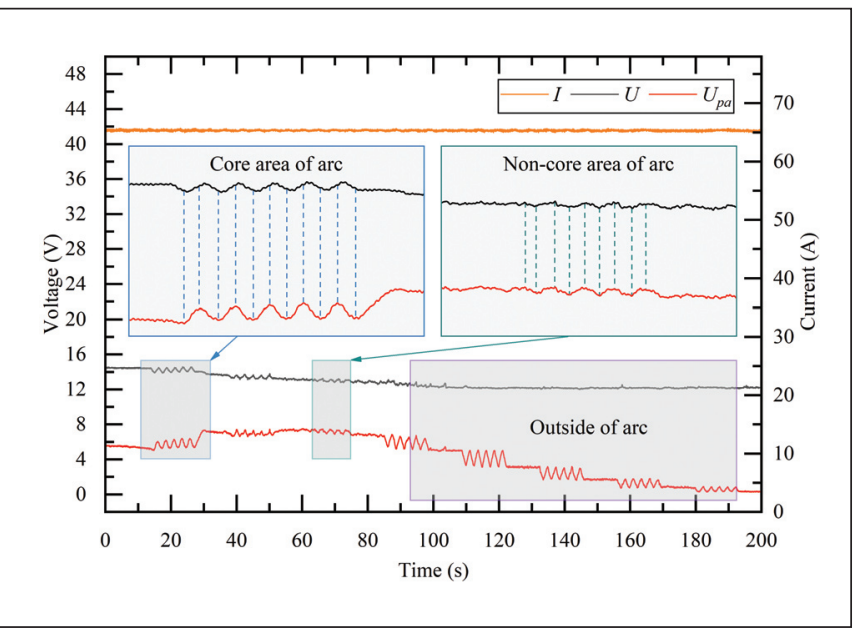

Fig. 9 - Electrical signals in Experiment \#3.

along with the arc voltage, was sufficient to reflect the related effects, so the follow up will only focus on probe voltage $U_{p a}$ to avoid repetition.

\section{Dynamic Effects}

In the actual welding process, the effects of the filler wire on the arc are more inclined to be dynamic effects. Figure 8 shows the electrical signals measured in Experiment \#2, where the end of the probe occupied the original core area of the arc. Because the arc plasma possessed heat capacity, it can be observed that if $\Delta T$ was too short (less than the time constant), $U_{p a}$ would not reach the stable level, and the fluctuation of $U_{p a}$ caused by the variation in $D$ would be more moderate with a decreasing $\Delta T$.

During the intermittent interference period (e.g., when $\Delta T$ was $4 \mathrm{~s}$ ), the local maximum of $U_{p a}$ did not appear when $U$ stayed at a lower level (when $D$ was $0.5 \mathrm{~mm}$ ), but approximately at a point when $U$ was rising (when $D$ was falling from 0.5 to $0 \mathrm{~mm}$ ), or probably when $U$ had just risen to the stable level 
(when $D$ had just completed the drop from 0.5 to $0 \mathrm{~mm}$ ). Similarly, the local minimum of $U_{p a}$ in Fig. 8 generally occurs when $U$ has just completed a drop, rather than when $U$ keeps at a higher level. The above-mentioned changes are mainly due to the variation in $D$ that can cause a sudden change in the conductive cross section, which can immediately change the current density and thus change electric field strength, while the arc itself has thermal inertia.

In the core area of the arc, the blocking by the probe tends to promote the ion recombination below the probe, while the ion recombination will lag behind the decrease in $D$ due to the thermal inertia. Thus, below the probe, the decrease in the electric field strength caused by the ion recombination will lag behind the decrease in $D$. On the contrary, the decrease in $D$ will lead to the sudden decrease in the conductive cross section, resulting in an immediate increase in electric field strength. Therefore, in the early stage of the decrease in $D, U_{p a}$ will rise because of the immediate increase in electric field strength caused by the sudden decrease in the conductive cross section. If $D$ has enough time to continue to decrease, the effect of ion recombination below the probe on the electric field strength will gradually emerge, thus reversing the growth in $U_{p a}$. Likewise, the gas ionization below the probe will also lag behind the increase in $D$, and $U_{p a}$ will change in the opposite direction. Therefore, the rate of ion recombination and the rate of gas ionization will affect the moments at which the local maximum and local minimum of the $U_{p a}$ occur, respectively. It can be observed that during the intermittent interference, the initial decline in $U_{p a}$ is steeper than its initial rise, which shows that the rate of ion recombination is faster than that of gas ionization in the early period after $D$ starts to vary. The same mechanism is also suitable for the continuous interference process. Thus, in Fig. $8, U_{p a}$ usually reaches the peak before $D$ falls to $0 \mathrm{~mm}$ and falls to the bottom when $D$ has just risen to $0.5 \mathrm{~mm}$.

Figure 9 shows the electrical signals measured in Experiment \#3 and that the dynamic probe continuously interfered with different areas of the arc column. It can also be seen that, in the core area of the arc, $U_{p q}$ can reach the local minimum before $U$ drops to the bottom. This is mainly because the change amount of $D$ becomes $1 \mathrm{~mm}$; it can rise to meet the moment that the decrease in $U_{p a}$ is reversed by the increase in local electric field strength caused by the gas ionization of the lower part of the arc. Therefore, when the continuous interference occurs in the original core area of the arc, an impression is presented that $U_{p a}$ does not strictly change synchronously with $U$, but often reaches peak or trough ahead of $U$. However, in the noncore area of the arc, the changes in $U_{p a}$ and $U$ seem to be more synchronous, and the dynamic relationship between them was more consistent with their static relationship because the effects of the probe on the arc were weakened. Additionally, the fluctuation of $U_{p a}$ became significant again outside the arc, which indicated that there is a large ionization gradient, so that the movement of probe can be detected particularly clearly. Although the droplet behavior was not covered here, the mechanism of its dynamic interference should also follow the above description.

\section{Conclusions}

This part of the work mainly studied the mechanism of the effects of the filler wire as a metal conductor on GTA based on the proposed arc-sensing method of detecting probe voltages. The main conclusions drawn are as follows:

1) When the filler wire as a metal conductor is fed into the arc, the arc voltage $(U)$ will rise due to the increase in the average electric field strength of the arc. The closer the filler wire is to the core area of the arc, the greater the $U$ will be.

2) The probe voltages $\left(U_{p a}\right.$ and $\left.U_{p c}\right)$ can reflect the electrical characteristics of different local areas of the GTA. The effects of the filler wire as a metal conductor on the different areas of the arc at different phases are not always consistent. When the filler wire is immersed in the original core area of the arc, the arc will be deflected, $U_{p a}$ will drop, and $U_{p c}$ will grow. When the filler wire is immersed in the original noncore area of the arc, the upper and lower parts of the arc will act as a whole, and the trend of $U_{p a}$ and $U_{p c}$ will follow that of $U$.

3) When the filler wire as a metal conductor causes dynamic reciprocating interference to the arc, the probe voltage does not strictly change synchronously with the arc voltage, but sometimes reaches peak or trough ahead of the arc voltage due to the thermal inertia. Nevertheless, the dynamic effects on the arc can indeed be reflected in the probe voltage.

\section{Acknowledgments}

This study is supported by the National Natural Science Foundation of China (Grant No.: 51505326), Natural Science Foundation of Tianjin (Grant No.: 16JCQNJC04300), and the Regional Demonstration Project of Marine Economic Innovation and Development (Grant No.: BHSF2017-10).

\section{References}

1. Chen, Z. Y., Chen, J., and Feng, Z. L. 2017. Monitoring weld pool surface and penetration using reversed electrode images. Welding Journal 96(10): 367-s to 375-s.

2. Fan, C. J., Lv, F. L., and Chen, S. B. 2009. Visual sensing and penetration control in aluminum alloy pulsed GTA welding. International Journal of Advanced Manufacturing Technology 42(1-2): 126-137. DOI: $10.1007 /$ s00170-008-1587-1

3. Song, H. S., and Zhang, Y. M. 2008. Measurement and analysis of three-dimensional specular gas tungsten arc weld pool surface. Welding Journal 87(4): 85-s to 95-s.

4. Zhang, W. J., Zhang, X., and Zhang, Y. M. 2013. Robust pattern recognition for measurement of three dimensional weld pool surface in GTAW. Journal of Intelligent Manufacturing 26(4): 659-676. DOI: 10.1007/s10845-013-0825-z

5. Zhang, W. J., Wang, X. W., and Zhang, Y. M. 2013. Analytical real-time measurement of a three-dimensional weld pool surface. Measurement Science and Technology 24(11): 115011. DOI: 10.1088/ 0957-0233/24/11/115011

6. Liu, Y. K., Zhang, Y. M., and Kvidahl, L. 2014. Skilled human welder intelligence modeling and control: Part 1 - Modeling. Welding Journal 93(2): 46-s to 52-s.

7. Dong, H., Cong, M., Zhang, Y. M., Liu, Y. K., and Chen, H. P. 2018. Modeling and real-time prediction for complex welding process based on weld pool. The International Journal of Advanced Manufacturing Technology 96(5-8): 2495-2508. DOI: 10.1007/ s00170-018-1685-7 
8. Cheng, Y. C., Xiao, J., Chen, S. J., and Zhang, Y. M. 2018. Intelligent penetration welding of thin-plate GTAW process based on arc voltage feedback. Transactions of the China Welding Institution 39(12): 1-4. DOI: 10.12073/j.hjxb.2018390287

9. Zhang, S. Q., Hu, S. S., and Wang, Z. J. 2016. Weld penetration sensing in pulsed gas tungsten arc welding based on arc voltage. Journal of Materials Processing Technology 229: 520-527. DOI: 10.1016/j.jmatprotec.2015.09.034

10. Renwick, R. J., Rarson, D. F., and Richardson, R. W. 1983. Experimental investigation of GTA weld pool oscillations. Welding Journal 62(2): 29-s to 35-s.

11. Xiao, Y. H., and Ouden, G. D. 1993. Weld pool oscillation during GTA welding of mild steel. Welding Journal 72(8): 428-s to 434-s.

12. Shi, Y., Li, C. K., Du, L. M., Gu, Y. F., and Zhu, M. 2016. Frequency characteristics of weld pool oscillation in pulsed gas tungsten arc welding. Journal of Manufacturing Processes 24: 145-151. DOI: 10.1016/j.jmapro.2016.08.010

13. Huang, J. K., Yang, M. H., Chen, J. S., Yang, F. Q., Zhang, Y. M., and Fan, D. 2018. The oscillation of stationary weld pool surface in the GTA welding. Journal of Materials Processing Technology 256: 57-68. DOI: 10.1016/j.jmatprotec.2018.01.018

14. Li, C. K., Shi, Y., Gu, Y. F., and Yuan, P. 2018. Monitoring weld pool oscillation using reflected laser pattern in gas tungsten arc welding. Journal of Materials Processing Technology 255: 876-885. DOI: 10.1016/j.jmatprotec.2018.01.037

15. Lv, N., and Chen, S. B. 2011. Investigation on acoustic signals for on-line monitoring of welding. Robotic Welding, Intelligence and Automation, Lecture Notes in Electrical Engineering, Springer, Berlin, Heidelberg, 88: 235-243.

16. Lv, N., Xu, Y. L., Zhong, J. Y., Chen, H. B., Wang, J. F., and Chen, S. B. 2013. Research on detection of welding penetration state during robotic GTAW process based on audible arc sound. Industrial Robot 40(5): 474-493.

17. Lv, N., Xu, Y. L., Fang, G., Zhao, H., and Chen, S. B. 2015. Mechanism analysis and feature extraction of arc sound channel for pulse GTAW welding dynamic process. Robotic Welding, Intelligence and Automation, RWIA 2014, Advances in Intelligent Systems and Computing, Springer International Publishing, Cham, 363: 249-261.

18. Zhang, Z. F., Wen, G. R., and Chen, S. B. 2018. Audible sound-based intelligent evaluation for aluminum alloy in robotic pulsed GTAW: Mechanism, feature selection and defect detection. IEEE Transactions on Industrial Informatics 14(7): 2973-2983. DOI: 10.1109/TII.2017.2775218

19. Yudodibroto, B. Y. B., Hermans, M. J. M., Hirata, Y., and den Ouden, G. 2004. Influence of filler wire addition on weld pool oscillation during gas tungsten arc welding. Science and Technology of Welding and Joining 9(2): 163-168. DOI: 10.1179/ 136217104225012274

20. Chen, S. J., Zhang, R. Y., Jiang, F., and Dong, S. W. 2018. Experimental study on electrical property of arc column in plasma arc welding. Journal of Manufacturing Processes 31: 823-832. DOI: 10.1016/j.jmapro.2017.12.028

21. Zhang, Y. M., Zhang, S. B., and Liu, Y. C. 2001. A plasma cloud charge sensor for pulse keyhole process control. Measurement Science and Technology 12(8): 1365-1370. DOI: 10.1088/09570233/12/8/352

22. Shan, P., Yi, X. L., Hu, S. S., and Luo, Z. 2003. Detection of plasma sheath voltage in plasma arc welding. Transactions of the China Welding Institution 24(2): 19-21.

SHUANGYANG ZOU, ZHIJIANG WANG (wangzj@tju.edu.cn), SHENGSUN HU, GUANCHENG ZHAO, and WANDONG WANG are with the Tianjin Key Laboratory of Advanced Joining Technology, School of Materials Science and Engineering, Tianjin University, Tianjin, China. YOUQUAN CHEN is with the Department of Mechanical Engineering, College of Humanities \& Information, Changchun University of Technology, Changchun, China.

\section{Welding Journal Now Publishing Direct Object Identifier (DOI) Numbers}

Dear members of the welding research community,

Note that in each issue of the Welding Journal Research Supplement, we are including Direct Object Identifier (DOI) numbers with each of the papers published in print and online. A direct object identifier is a unique alphanumeric string assigned by a registration agency (we are using Crossref.org) to identify content and provide a persistent link to its location on the Internet. Our decision to begin assigning a DOI for each paper comes directly from a request by the research community.

As part of our obligation to Crossref.org, we are asked to provide DOI numbers, when available, in the references section of papers. So, if you have submitted a paper to the Welding Journal or are planning on submitting a paper, we ask that you update your references to include DOI numbers whenever possible.

Thank you.

Annette Alonso

Publisher, Welding Journal 